\section{Plant Growth Stimulation and Root Colonization Potential of In Vivo versus In Vitro Arbuscular Mycorrhizal Inocula}

\author{
Cinta Calvet ${ }^{1}$, Amelia Camprubi, and Ana Pérez-Hernández \\ Institut de Recerca i Tecnologia Agroalimentàries (IRTA), Patologia Vegetal, \\ Ctra. de Cabrils Km 2, E-08348 Cabrils, Barcelona, Spain
}

\author{
Paulo Emilio Lovato \\ Depto. de Engenharia Rural-CCA, Universidade Federal de Santa Catarina, \\ CP 476, 88040-970 Florianópolis, Brazil
}

Additional index words. inoculum production, biofertilizer, spore morphology, Glomus intraradices, Rhizophagus irregularis, root organ culture

\begin{abstract}
Inoculum of arbuscular mycorrhizal fungi, with growing use in horticulture, is produced mainly in two technically different cultivation systems: in vivo culture in symbiosis with living host plants or in vitro culture in which the fungus life cycle develops in association with transformed roots. To evaluate the effectiveness and the infectivity of a defined isolate obtained by both production methods, a replicated comparative evaluation experiment was designed using different propagules of Rhizophagus irregularis produced in vivo on leek plants or in vitro in monoxenic culture on transformed carrot roots. The size of the spores obtained under both cultivation methods was first assessed and bulk inoculum, spores, sievings, and mycorrhizal root fragments were used to inoculate leek plantlets. Spores produced in vitro were significantly smaller than those produced in vivo. Although all mycorrhizal propagules used as a source of inoculum were able to colonize plants, in all cases, leek plants inoculated with propagules obtained in vivo achieved significantly higher mycorrhizal colonization rates than plants inoculated with in vitro inocula. Inoculation with in vivo bulk inoculum and in vivo mycorrhizal root fragments were the only treatments increasing plant growth. These results indicate that the production system of arbuscular mycorrhizal fungi itself can have implications in the stimulation of plant growth and in experimental results.
\end{abstract}

Arbuscular mycorrhizas (AM) are the most abundant microbial symbiosis for the majority of plant species, improving their nutrition and fitness. The presence of this mutualistic association in natural and agroecosystems, and especially the role of the fungal partner at increasing crop yields and plant health, has stimulated the search for mass production methods. Arbuscular mycorrhizal fungi (AMF) are unable to complete their life cycle and reproduce apart from the host plants, although their spores can germinate under different environmental conditions in the absence of a host (Giovannetti, 2001). Inoculum production has been improved in the last decades, but a recent article by Vosátka et al. (2012)

\footnotetext{
Received for publication 8 Apr. 2013. Accepted for publication 22 May 2013.

We thank Dr. Christopher Walker for helpful advice on the taxonomy of the arbuscular mycorrhizas fungal isolate used in this work. We acknowledge financial support from the Spanish Ministry of Economy and Competitiveness MINECO grant AGL2010-15017. Paulo E. Lovato had a fellowship from CAPES Foundation, Ministry of Education of Brazil, Brasília/DF-Brazil.

${ }^{1}$ To whom reprint requests should be addressed; e-mail cinta.calvet@irta.cat.
}

confirms that commercial inocula sold on international markets are not always able to perform the arbuscular mycorrhizal symbiosis. There are basically three production systems for commercially available inoculants, including a variety of fungal isolates, formulations, and components: the substratebased production system, the substrate-free cultivation system (both under in vivo conditions), and the in vitro cultivation system (Ijdo et al., 2011). The in vivo substrate-based cultivation of AM in container-grown plants is the traditional and the most widely used technique for AM fungal inoculum production. It is useful for large-scale production that requires little technical support. The in vivo substrate-free system is based on hydroponics and aeroponics, but it has been limited to a few fungal species. The in vitro system allows obtaining sterile propagules with high potential for research and high-quality inoculum while lacking other microorganisms. This method has been widely tested and adopted by mycorrhizal research laboratories around the world (Declerck et al., 2005). Several reviews describe the methods for large-scale production of AMF and point out their potentials and limitations (Adholeya et al., 2005; Declerck et al., 2005; Diop, 2003; Elmes and Mosse, 1984; Ferguson and Woodhead, 1982;
Fortin et al., 2002; Gianinazzi et al., 2002; Ijdo et al., 2011).

Some studies on the intraradical AM development in monoxenic roots suggest characteristics of internal colonization with similar structures to those of in vivo soil-grown plants (Bago and Cano, 2005), but direct comparisons of host plant responses to these two types of inoculum are not found in the literature. In the present work, inocula of a defined isolate (BEG 72) of Rhizophagus irregularis simultaneously produced, for equal time, in vivo and in vitro, were evaluated in terms of host root colonization potential and plant growth stimulation.

\section{Materials and Methods}

The fungus used, obtained from Citrus aurantium L. (Camprubi and Calvet, 1996) in northeastern Spain, was isolate BEG 72 (International Bank of Glomeromycota) of Rhizophagus irregularis (Blaszk., Wubet, Renker \& Buscot) C Walker \& A. Shüssler comb nov according to $500 \mathrm{bp}$ LSU sequence analysis (Camprubi et al., 2008) and formerly known as Glomus intraradices (Krüger et al., 2012; Stockinger et al., 2009). It was simultaneously cultivated during 6 months in association with leek (Allium porrum L., "carentan 2") plants and in monoxenic culture on Ri T-DNA (plasmid of Agrobacterium rhizogenes) transformed carrot (Daucus carota L.) roots (Mugnier and Mosse, 1987).

For in vivo inoculum production, leek plants inoculated with $R$. irregularis were grown in 1-L volume containers filled with Terragreen (Oil-Dri Company, Wisbech, U.K.) for 6 months in a greenhouse under sodiumvapor lamps (temperature 20 to $28^{\circ} \mathrm{C}$ and $\left.135 \mu \mathrm{mol} \cdot \mathrm{s}^{-1} \cdot \mathrm{m}^{-2} 16 \mathrm{~h} \cdot \mathrm{d}^{-1}\right)$. In vitro production consisted of monoxenic cultures of the same fungus established in bicompartmented petri plates supporting fungal development on modified Strullu-Romand medium (MSR) in the root compartment and on MSR medium without sucrose or vitamins in the root-free compartment (Declerck et al., 1996; St. Arnaud et al., 1996). Plates were incubated at $25^{\circ} \mathrm{C}$ for the same duration, i.e., during 6 months. To assure that similar material was used, we assessed mycorrhizal colonization in both the host plant root systems and the monoxenically grown roots after 6 months by the grid-line intersect method (Giovannetti and Mosse, 1980).

Aiming to test a possible influence of the production method, we assessed spore diameter size, because previous observations on spore morphology indicated possible differences between spores produced in vitro and in vivo. Individual spores were extracted from the soil rhizosphere of a 6-month-old leek plant culture by wet sieving (250-, 125-, and $50-\mu \mathrm{m}$ mesh) and decanting (Gerdemann and Nicolson, 1963) and also from the rootfree compartments of four petri plates with 6-month-old monoxenic cultures after liquefying the medium with sodium citrate buffer (Doner and Bécard, 1991). One hundred spores from each origin were included in an acid 
glycerol solution $(90 \mathrm{~mL}$ distilled water, 10 $\mathrm{mL} 1 \% \mathrm{HCl}, 100 \mathrm{~mL}$ glycerin) individually placed on a glass slide and measured under a compound microscope. Two perpendicular transversal measures were recorded for each spore and their arithmetic mean value was the variable used in statistical analysis. A oneway analysis of variance $(P \leq 0.05)$ indicated no significant differences among the size of spores produced in the four petri plates and because differences resulting from the monoxenic cultures themselves could be discarded, we compared a single sample of 100 spores produced in vitro with the sample of spores produced in association with a living plant.

We carried out a comparison experiment to evaluate the influence of both inocula production methods on their effectiveness in root colonization and growth stimulation of leek plants. The experiment was replicated within a 4-month interval and plant growth was monitored at the end of the second experiment. Mycorrhizal leek plants and the four monoxenically cultured petri plates mentioned were used as inoculum sources for the experimental setup. Because preliminary analyses showed that the mycorrhizal colonization in the roots of both the leek plant culture and the $\mathrm{Ri} \mathrm{T}$-DNA transformed carrot roots in the in vitro cultures was similar and above $80 \%$, we sought to apply equivalent amounts of potential mycorrhizal propagules originated in vivo and in vitro, and inoculation treatments were as follows.

In vivo inoculation treatments consisted of: 1) bulk inoculum: $2 \mathrm{~mL}$ of substrate (with $\approx 50 \%$ pore space) from the host plant rhizosphere, which contained $50 \pm 15$ spores/g and $155 \pm 54 \mathrm{~mm}$ of root fragments varying in shape and size (Newman, 1966) plus external mycelium fragments; 2) roots: $1010-\mathrm{mm}$ root fragments excised from the host plant heavily colonized root system; 3) spores: 50 individual spores recovered by wet sieving (pool of all spores retained on 250-, 125-, and 50- $\mu \mathrm{m}$ mesh sieves) of the bulk inoculum; and 4) sieving: $20-\mathrm{mL}$ aliquot of a distilled water suspension of fungal material recovered after sieving a 2-mL sample of in vivo bulk inoculum through a $125-\mu \mathrm{m}$ mesh sieve.

In vitro inoculation treatments consisted of: 1) bulk inoculum: 1-mL portions excised from the MSR medium root compartment, which contained $70 \pm 21$ spores and $284 \pm$ $83 \mathrm{~mm}$ of heavily colonized root fragments varying in shape and size (Newman, 1966) plus external fungal mycelium; 2) roots: 10 densely colonized mycorrhizal $10-\mathrm{mm}$ root fragments excised from the same compartment after accurate observation under a stereomicroscope ( $\times 80$ magnification) to confirm mycorrhizal colonization; 3) spores: 50 individual spores obtained after liquefying the medium in the root-free compartment with sodium citrate buffer as mentioned previously; and 4) sieving: 20-mL aliquot of a distilled water suspension including fungal material recovered after sieving through $125 \mu \mathrm{m}$ a suspension obtained after dissolving the agar from the root-free compartment of a monoxenically cultured petri plate.
A noninoculated control treatment was included in the experimental design. To verify possible nonmycorrhizal effects of other inoculum components on plant growth, after harvesting Expt. 2, four additional control treatments were compared with the former control treatment: $20-\mathrm{mL}$ leachates free of mycorrhizal propagules from in vivo and in vitro bulk inoculum water suspensions, nonmycorrhizal leek roots, and nonmycorrhizal transformed carrot root fragments.

To estimate the colonization density in roots, the number of propagules in $2010-\mathrm{mm}$ root fragments from a 6-month-old leek culture in Terragreen and from a 6-month-old in vitro culture of $R$. irregularis (BEG 72) were counted under the stereomicroscope $(\times 80$ magnification). Plant root samples were previously cleared and stained (Koske and Gemma, 1989), whereas structures inside in vitro roots, visible without any processing, were observed and counted with no clearing nor staining.

Leek plantlets previously germinated in autoclaved sand trays were transplanted to $100-\mathrm{mL}$ individual containers filled with pasteurized $\left(100{ }^{\circ} \mathrm{C}, 2 \mathrm{~h}\right.$, twice) sandy soil (950 $\mathrm{g} \cdot \mathrm{kg}^{-1}$ sand, less than $2.5 \mathrm{~g} \cdot \mathrm{kg}^{-1}$ organic matter, $\mathrm{pH} 8.2,2.0 \mathrm{mg} \cdot \mathrm{kg}^{-1}$ Olsen-P), and the different inoculation treatments were applied under the plant roots at transplant. Containers, kept for 10 weeks in a greenhouse (20 to $28{ }^{\circ} \mathrm{C}$ and $135 \mu \mathrm{mol} \cdot \mathrm{s}^{-1} \cdot \mathrm{m}^{-2} 16 \mathrm{~h} \cdot \mathrm{d}^{-1}$ ), received weekly applications of Hoagland's solution without phosphorus and irrigation was controlled daily. There were six replicates for each treatment and we carried out two consecutive experiments within a 4-month period. The percentage of root colonization was estimated (Giovannetti and Mosse, 1980) for both subsequent experiments and they were shown to be reproducible. When mycorrhizal root colonization percentage data obtained in each replicated experiment were analyzed as a factorial design, no significant differences $(P \leq 0.05)$ between them could be detected. Therefore, 12 replications per inoculation treatment in a single experimental design were considered for statistical analysis in two one-way analyses of variance (ANOVAs) $(P \leq 0.05)$, comparing first the origin of the inocula (either in vivo or in vitro) and second the type of propagules used as an inoculum source. At the end of the second experiment, plant shoot dry weight was determined. Results were statistically analyzed by ANOVA and means were compared with Tukey test $(P \leq 0.05)$.

\section{Results}

Figure 1 shows the spore size distribution of $100 \mathrm{R}$. irregularis spores obtained in monoxenic culture in petri plates or in host plant culture in the greenhouse. Spores had a similar morphological shape regardless of their origin, but the size of the spores produced in vitro was significantly smaller $(P \leq$ $0.05)$. Spores produced in vitro had a mean diameter of $83 \pm 9 \mu \mathrm{m}$, whereas those produced in vivo had a mean diameter of $117 \pm$ $13 \mu \mathrm{m}$, and spore size distribution indicated clear variations between both samples. The diameter of spores produced in vitro varied between $66 \mu \mathrm{m}$ and $105 \mu \mathrm{m}$ with the highest frequencies between 70 and $89 \mu \mathrm{m}$, whereas the in vivo spore diameters ranged between $90 \mu \mathrm{m}$ and $145 \mu \mathrm{m}$ with the highest frequencies between 100 and $134 \mu \mathrm{m}$. Spores produced in vitro were less pigmented and more homogenous in size and color. Concerning the colonization density in roots, in plant root fragments, there were $88 \pm 45$ intraradical vesicles/cm, whereas in vitro roots had $35 \pm$ 23 spores $/ \mathrm{cm}$.

After a 10 -week growth period in a greenhouse, leek plants inoculated with the in vivo treatments had higher mycorrhizal colonization rates in roots than the equivalent in vitro

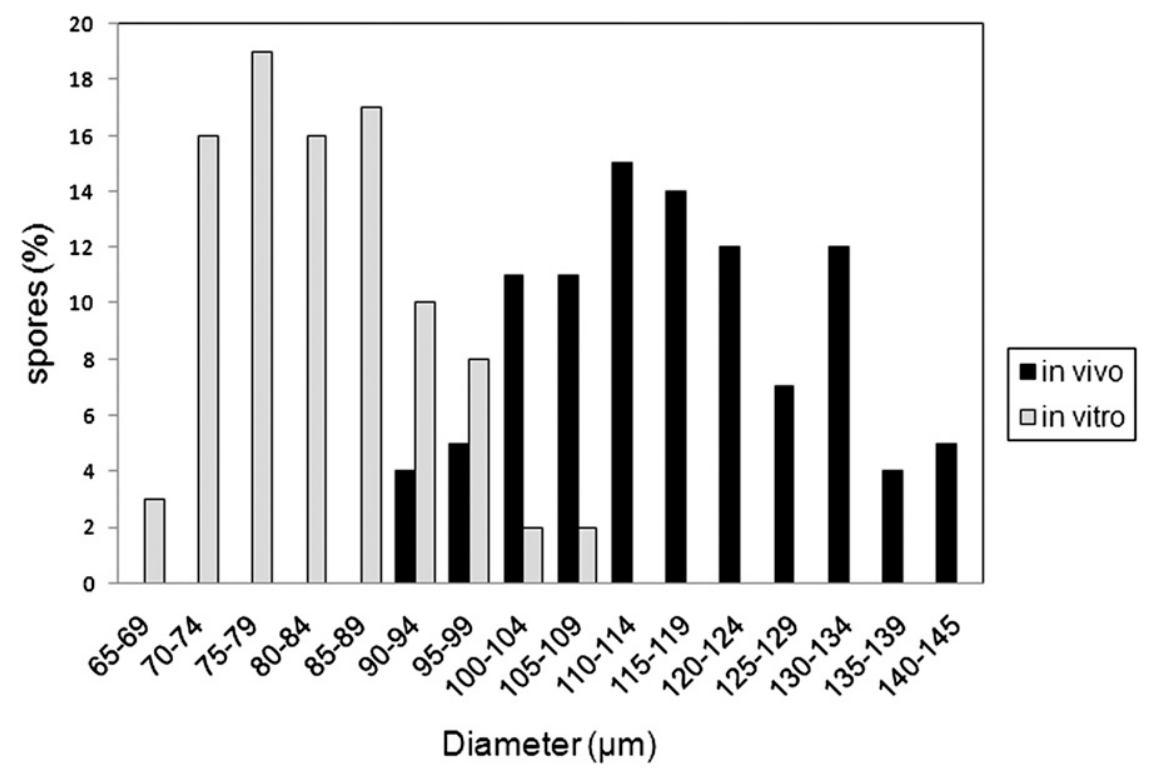

Fig. 1. Distribution of diameter values of two populations of 100 Rhizophagus irregularis spores recovered from in vivo or in vitro cultures. 
inoculation treatments (Fig. 2A). Among the in vivo treatments, bulk inoculum, root fragments, and sievings were significantly more efficient than individual spores at colonizing the root system, although only bulk inoculum and root fragments infected all the inoculated plants (12). When $125-\mu \mathrm{m}$ sievings were used as an in vivo inoculum source, 11 of 12 leek plants were mycorrhizal, whereas only nine of 12 plants became infected in the inoculation treatment with individual spores. Among in vitro propagules, bulk inoculum was the sole in vitro inoculum source able to colonize all 12 inoculated plants. Nine plants became mycorrhizal when inoculated with in vitro obtained root fragments or spores, and only seven were infected after inoculation with $125-\mu \mathrm{m}$ sievings.

Plant growth measured as shoot dry weight showed a response only to in vivo-produced inocula. None of the inoculation treatments based on in vitro-obtained propagules stimulated plant growth when compared with the noninoculated control (Fig. 2B), and no differences were detected among them either.
Among in vivo-obtained propagules, mycorrhizal root fragments were the most efficient at stimulating shoot development followed by the bulk inoculum treatment. They both significantly increased the growth of leek plants when compared with the noninoculated control (Fig. 2B). The application of nonmycorrhizal inoculum leachates and of nonmycorrhizal root fragments had no influence on plant growth because no significant differences could be reported when these treatments were compared with the noninoculated control treatment, showing that all differences in plant growth were the result of the mycorrhiza.

\section{Discussion}

The significantly different sizes of $R$. irregularis BEG 72 spores produced in either in vitro or in vivo systems indicate distinct adaptations of the fungus to the growth medium. Pawlowska et al. (1999) also observed that spores of Glomus etunicatum Becker \& Gerdemann from monoxenic cultures exhibited

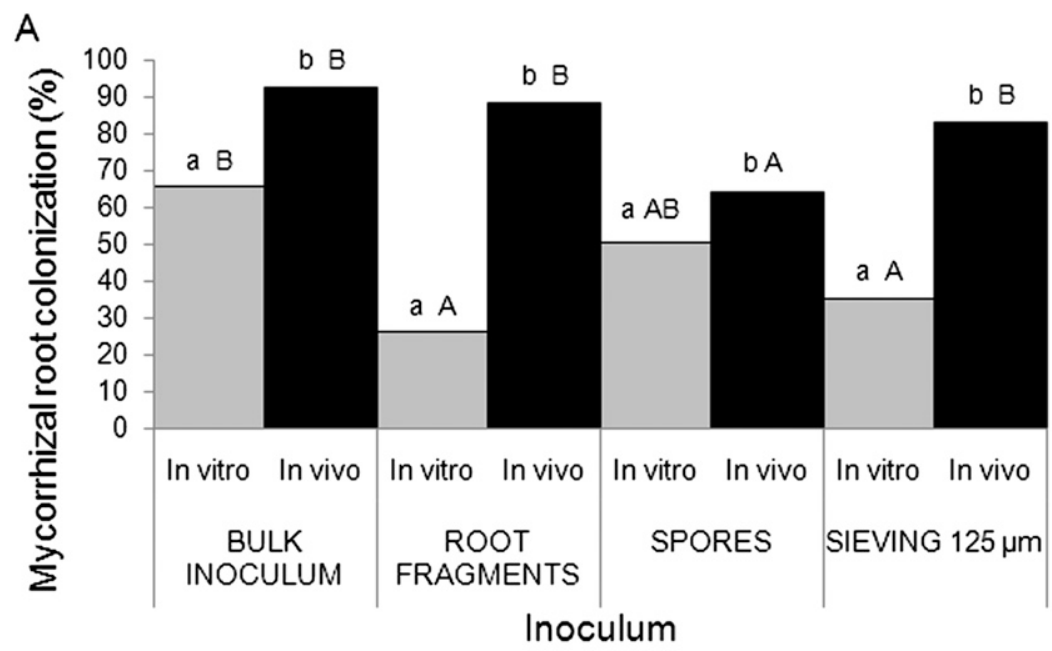

\section{B}

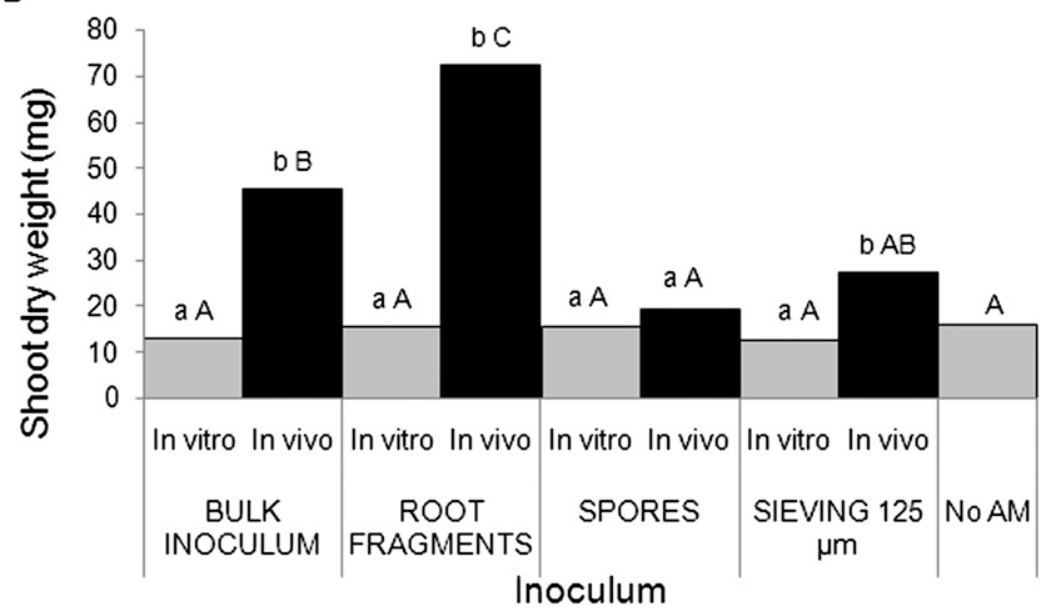

Fig. 2. Mycorrhizal root colonization (A) and growth of "carentan 2" leek plants (B) inoculated with bulk inoculum, root fragments, spores, and inoculum sievings from monoxenic culture (in vitro) or from plant culture in association with a host plant (in vivo). Different lower case letters indicate significant differences between in vivo and in vitro inoculation treatments (Tukey $P \leq 0.05$ ). Different upper case letters indicate significant differences among in vitro inocula or among in vivo inocula (Tukey $P \leq$ 0.05). Data are means of 12 plants (A) and six plants (B).

smaller diameters than soilborne spores; the mean diameter value of mature G. etunicatum spores formed in vitro was $96 \mu \mathrm{m}$, whereas the mean diameter of spores recovered from pot cultures measured $106 \mu \mathrm{m}$. In the present work, the differences found in $R$. irregularis spore size were greater with a mean increase of $34 \mu \mathrm{m}$ in spores obtained from plant culture, corresponding to a $40 \%$ gain. One possible reason for the smaller size of in vitroproduced spores is depletion of nutrients or carbon in the root organ culture. Previous work has shown that replenishing carbon or nutrients can increase spore number (Douds, 2002), but information on spore size was not provided. Spore size has shown a linear relation to its number of nuclei (Marleau et al., 2011), a characteristic that hypothetically could be linked to the potential of the fungus to develop after germination and to establish the association with the plant. We observed that in vitro spores were, in general, of lighter color, confirming differences in color intensity resulting from the production system, as reported by Fortin et al. (2002).

All in vivo-produced inoculum treatments achieved higher colonization rates as compared with inocula produced under in vitro conditions using Ri T-DNA transformed carrot roots. Among inocula produced in vitro, bulk inoculum, which included all kinds of propagules: spores, hyphae, and root segments, achieved the highest colonization rate in leek roots after 10 weeks (above 60\%). Nevertheless, it was unable to enhance plant growth, like all the other in vitro treatments (Fig. 2B). Bulk inoculum and mycorrhizal root fragments produced in vivo were the only treatments capable of significantly stimulating plant growth.

In vivo mycorrhizal inoculation treatments had high infectivity potentials, whereas the equivalent in vitro treatments were less effective at colonizing the plant's root system. Although all types of propagules of AM fungi (spores, root fragments, and hyphae) are able to initiate AM symbiosis (Mosse, 1988), the inoculation treatments based on the use of spores alone were not reliable because only nine of 12 plants became mycorrhizal, and sievings also lacked infectivity. A leaching effect in the leek test plant sandy soil containers could have caused a loss in the number of spores and mycelial fragments and an indirect decrease of the infectivity potential when they were used as the sole inoculum source. Vimard et al. (1999) also observed a drastic reduction in plant colonization when in vitro-produced spores were used in a highly porous substrate as compared with the colonization by spores in soil or by root fragments either in soil or in the porous substrate.

High propagule density reduces the length of the lag phase in the curve of colonization percentage vs. time, and that is related to a quick spread of the fungus in the root system (Smith and Read, 1997; Vimard et al., 1999). The increases in colonization and in plant growth stimulation when root fragments from a pot culture were used as inoculum might thus be related to the high number of infective 
intraradical vesicles of $R$. irregularis, a morphological characteristic of the species when produced in vivo. Fortin et al. (2002) showed that arbuscules and vesicles are often scarce in mycorrhizal Ri T-DNA transformed carrot roots despite the production of abundant intracortical mycelium, suggesting a delay in the development of the symbiosis when they are used as inoculum. In our experimental conditions, the number of spores observed on in vitro root fragments $(35 \pm 23$ per $\mathrm{cm})$, even if seemingly lower than the number of vesicles counted within in vivo roots $(88 \pm 46$ per $\mathrm{cm})$, was expected to have an infectivity potential similar to that of in vivo root fragments. However, root colonization achieved by in vivo root fragments when used as inoculum was much higher despite the uncertain density of propagules within the plant roots effectively used as inocula. Because internal colonization cannot be accurately assessed without a staining procedure, their mycorrhizal potential is assumed to be equivalent to the colonization estimated in stained samples from part of the root system.

The propagules originating from inside in vivo root fragments, in this case spores, vesicles, or hyphae, must have colonized the host root earlier, therefore having stimulated the host growth by the time plant biomass was measured, which was 10 weeks. On the other hand, propagules from in vitro inoculants probably promoted a later colonization of roots with the resulting lack of response in plant growth. This hypothesis is supported by the delay in early plant colonization observed by Vimard et al. (1999), when in vitro spores were compared with living mycorrhizal plant roots as an inoculum source. After 16 weeks, levels of colonization achieved by living roots were still significantly greater than those achieved by in vitro spores, but plant growth response was not evaluated in that work.

Loss of infectivity along generations of inocula produced in vitro, both for spores and mycorrhizal root pieces, might reduce the infectivity and the effectiveness of the subcultures (Plenchette et al., 1996). This has been abundantly reported for pathogenic fungi (Agrios, 2005; Butt et al., 2006; Krokene and Solheim, 2001; Skovgaard et al., 2002), which show lower virulence after several monoxenic in vitro culture cycles and also for ectomycorrhizal fungi (Marx and Daniel, 1976). Because we used a first-cycle subculture of Ri T-DNA transformed carrot roots infected with the AMF isolate, the lower infectivity of these inoculants could not be the result of an aging effect of in vitro subcultures, therefore seeming to be a trait of the fungus when grown in root organ cultures.

Another hypothesis is the effect of bacteria associated with the AMF spores, which can be beneficial to the association (Barea et al., 2005). It has been demonstrated that these bacteria and AMF have beneficial effects on each other (Bharadwaj et al., 2012; Lioussanne et al., 2010) and that such effects depend on the bacterial and fungal partners involved, being similar across plant species (Pivato et al., 2009). Because only bulk inoculum and mycorrhizal root fragments were able to increase plant growth, one possible hypothesis is that benefits to plant growth depend on agents or factors present inside plant roots, one of such factors being bacteria associated with AMF. Continuous in vitro culture of one species of Gigaspora led to the elimination of the endobacteria from its large spores (Bianciotto et al., 2004). Such bacteria are important for the fungus development (see review by Bonfante and Anca, 2009), because they affect hyphal elongation and branching and have been found in several AMF species (Lumini et al., 2007). Rhizophagus irregularis forms smaller intraradical spores, and the loss of bacteria when cultured in vitro might be linked to changes in the symbiotic process.

The reasons for the phenomena we observed led to several hypotheses indicating fascinating avenues for fundamental research. Our main concern, however, was to control if propagules of the same AMF produced in vitro were similar to those obtained from the classical plant culture system.

The in vitro systems have been proved useful for the cultivation and conservation of a large number of species and isolates of AM fungi (Fortin et al., 2002), being a valuable tool to study fundamental aspects of AM symbiosis. They are also a very good system for massive production of uncontaminated spores and have been adopted both for research and for commercial purposes. Nevertheless, the diverging behaviors in colonization and in growth effects of in vivo and in vitro inocula must be taken into account when extrapolating research results obtained with monoxenically cultured AM fungi to plant cultured AM fungi. Diop (2003) stated that the effectiveness of these propagules in natural conditions should be investigated, and our results confirm the need to still increase our knowledge on dynamics of colonization and of exchanges between AM fungi and host plants concerning the inocula origin. The data reported here indicate that the production system of AM fungi itself can have implications in experimental results, thus requiring thorough investigation of mechanisms involved as well as monitoring of responses in cropping systems.

\section{Literature Cited}

Adholeya, A., P. Tiwari, and R. Singh. 2005. Largescale production of arbuscular-mycorrhizal fungi on organs and inoculation strategies, $p$. 315-338. In: Declerck, S., D.G. Strullu, and J.A. Fortin (eds.). In vitro culture of mycorrhizas. Springer-Verlag, Berlin, Heidelberg, Germany.

Agrios, G.N. 2005. Plant pathology. 5th Ed. Academic Press, San Diego, CA.

Bago, A. and C. Cano. 2005. Breaking myths on arbuscular mycorrhizas in vitro biology, p. 111138. In: Declerck, S., D.G. Strullu, and J.A. Fortin (eds.). In vitro culture of mycorrhizas. Springer-Verlag, Berlin, Heidelberg, Germany.

Barea, J.M., M.J. Pozo, R. Azcon, and C. AzconAguilar. 2005. Microbial co-operation in the rhizosphere. J. Exp. Biol. 56:1761-1778.

Bharadwaj, D.P., S. Alstrom, and P.O. Lundquist. 2012. Interactions among Glomus irregulare, arbuscular mycorrhizal spore-associated bacteria, and plant pathogens under in vitro conditions. Mycorrhiza 22:437-447.

Bianciotto, V., A. Genre, P. Jargeat, E. Lumini, G. Bécard, and P. Bonfante. 2004. Vertical transmission of endobacteria in the arbuscular mycorrhizal fungus Gigaspora margarita through generation of vegetative spores. Appl. Environ. Microbiol. 70:3600-3608.

Bonfante, P. and I.A. Anca. 2009. Plants, mycorrhizal fungi, and bacteria: A network of interactions. Annu. Rev. Microbiol. 63:363-383.

Butt, T.M., C. Wang, A.A. Shah, and R. Hall. 2006. Degeneration of entomogenous fungi, p. 213226. In: Eilenbergand, J. and H.M.T. Hokkanen (eds.). An ecological and societal approach to biological control. Springer-Verlag, Heidelberg, Germany.

Camprubi, A. and C. Calvet. 1996. Isolation and screening of mycorrhizal fungi from citrus nurseries and orchards and inoculation studies. HortScience 31:366-369.

Camprubi, A., V. Estaun, A. Nogales, F. GarciaFigueres, M. Pitet, and C. Calvet. 2008. Response of the grapevine rootstock Richter 110 to inoculation with native and selected arbuscular mycorrhizal fungi and growth performance in a replant vineyard. Mycorrhiza 18: 211-216.

Declerck, S., D.G. Strullu, and J.A. Fortin. 2005. In vitro culture of mycorrhizas. Springer-Verlag, Berlin, Heidelberg, Germany.

Declerck, S., D.G. Strullu, and C. Plenchette. 1996. In vitro mass production of arbuscular mycorrhizal fungus Glomus versiforme associated with Ri T-DNA transformed carrot roots. Mycol. Res. 100:1237-1242.

Diop, T.A. 2003. In vitro culture of arbuscular mycorrhizal fungi: Advances and future prospects. Afr. J. Biotechnol. 2:692-697.

Doner, L.W. and G. Bécard. 1991. Solubilization of gellan gels by chelation of cations. Biotechnol. Tech. 5:25-28.

Douds, D.D. 2002. Increased spore production by Glomus intraradices in the split-plate monoxenic culture system by repeated harvest, gel replacement, and resupply of glucose to the mycorrhizal. Mycorrhiza 12:163-167.

Elmes, R.P. and B. Mosse. 1984. Vesicular-arbuscular endomycorrhizal inoculum production. II. Experiments with maize (Zea mays) and other hosts in nutrient flow culture. Can. J. Bot. 62:15311536.

Ferguson, J.J. and S.H. Woodhead. 1982. Production of endomycorrhizal inoculum, p. 47-54. In: Schenck, N.C. (ed.). Methods and principles of mycorrhizal research. Amer. Phytopathol. Soc., St. Paul, MN.

Fortin, J.A., G. Bécard, S. Declerck, Y. Dalpé, M. St-Arnaud, P. Coughlan, and Y. Piché. 2002. Arbuscular mycorrhiza on root-organ cultures. Can. J. Bot. 80:1-20.

Gerdemann, J.W. and T.H. Nicolson. 1963. Spores of mycorrhizal Endogone extracted from soil by wet sieving and decanting. Trans. Br. Mycol. Soc. 46:235-244.

Gianinazzi, S., H. Schüepp, J.M. Barea, and K. Haselwandter. 2002. Mycorrhizal technology in agriculture: From genes to bioproducts. Birkhauser, Basel, Switzerland.

Giovannetti, M. 2001. Survival strategies in arbuscular mycorrhizal symbionts, p. 185-196. In: Seckbach, J. (ed.). Symbiosis mechanisms and model systems. Academic Publisher, Dordrecht, The Netherlands.

Giovannetti, M. and B. Mosse. 1980. An evaluation of techniques for measuring vesicular-arbuscular mycorrhizal infection in roots. New Phytol. 87:489-500. 
Ijdo, M., S. Cranenbrouck, and S. Declerck. 2011. Methods for large-scale production of AM fungi: Past, present and future. Mycorrhiza 21: $1-16$.

Koske, R.E. and J.N. Gemma. 1989. A modified procedure for staining roots to detect VA mycorrhizas. Mycol. Res. 92:486-488.

Krokene, P. and H. Solheim. 2001. Loss of pathogenicity in the blue-stain fungus Ceratocystis polonica. Plant Pathol. 50:497-502.

Krüger, M., C. Krüger, C. Walker, H. Stockinger, and A. Schüßler. 2012. Phylogenetic reference data for systematics and phylotaxonomy of arbuscular mycorrhizal fungi from phylum to species level. New Phytol. 193:970-984.

Lioussanne, L. 2010. The role of the arbuscular mycorrhiza-associated rhizobacteria in the biocontrol of soilborne phytopathogens. Span. J. Agr. Res. 8:S51-S61.

Lumini, E., V. Bianciotto, P. Jargeat, M. Novero, A. Salvioli, G. Becard, and P. Bonfante. 2007. Presymbiotic growth and sporal morphology are affected in the arbuscular mycorrhizal fungus Gigaspora margarita cured of its endobacteria. Cell. Microbiol. 9:1716-1729.

Marleau, J., Y. Dalpé, M. St.-Arnaud, and M. Hijri. 2011. Spore development and nuclear inheritance in arbuscular mycorrhizal fungi. BMC Evol. Biol. 11:51-62.
Marx, D.H. and W.J. Daniel. 1976. Maintaining cultures of ectomycorrhizal and plant pathogenic fungi in sterile cold water storage. Can. J. Microbiol. 22:338-341.

Mosse, B. 1988. Some studies relating to 'independent' growth of vesicular-arbuscular endophytes. Can. J. Bot. 66:2533-2540.

Mugnier, J. and B. Mosse. 1987. Vesicular-arbuscular infections in Ri T-DNA transformed roots grown monoxenically. Phytopathology 77:1045-1050.

Newman, E.I. 1966. A method of estimating the total length of root in a sample. J. Appl. Ecol. 3:139-145.

Pawlowska, T., D.D. Douds, and I. Charvat. 1999. In vitro propagation and life cycle of the arbuscular mycorrhizal fungus Glomus etunicatum. Mycol. Res. 103:1549-1556.

Pivato, B., P. Offre, S. Marchelli, B. Barbonaglia, C. Mougel, P. Lemanceau, and G. Berta. 2009. Bacterial effects on arbuscular mycorrhizal fungi and mycorrhiza development as influenced by the bacteria, fungi, and host plant. Mycorrhiza 19:81-90.

Plenchette, C., S. Declerck, T.A. Diop, and D.G. Strullu. 1996. Infectivity of monoxenic subcultures of the arbuscular mycorrhizal fungus Glomus versiforme associated with Ri-T-DNAtransformed carrot root. Appl. Microbiol. Biotechnol. 46:545-548.
Skovgaard, K., L. Bodker, and S. Rosendahl. 2002. Population structure and pathogenicity of members of the Fusarium oxysporum complex isolated from soil and root necrosis of pea (Pisum sativum L.). FEMS Microbiol. Ecol. 42:367-374.

Smith, S.E. and D.J. Read. 1997. Mycorrhizal symbiosis. Academic Press, San Diego, CA.

St. Arnaud, M., C. Hamel, B. Vimard, M. Caron, and J.A. Fortin. 1996. Enhanced hyphal growth and spore production of the arbuscular mycorrhizal fungus Glomus intraradices in an in vitro system in the absence of host roots. Mycol. Res. 100:328-332.

Stockinger, H., C. Walker, and A. Schüßler. 2009. Glomus intraradices DAOM197198, a model fungus in arbuscular mycorrhiza research, is not Glomus intraradices. New Phytol. 183: 1176-1187.

Vimard, B., M. St.-Arnaud, V. Furlan, and J.A. Fortin. 1999. Colonization potential of in vitroproduced arbuscular mycorrhizal fungus spores compared with a root-segment inoculum from open pot culture. Mycorrhiza 8:335-338.

Vosátka, M., A. Látr, S. Gianinazzi, and J. Albrechtová. 2012. Development of arbuscular mycorrhizal biotechnology and industry: Current achievements and bottlenecks. Symbiosis 58:29-37. 